\section{AGROECOLOGY}

\section{Feed the world and keep the trees}

A worldwide switch to vegetarian diets could allow the planet's estimated 2050 population of 9.7 billion to feed themselves without cutting down any more forests.

Karl-Heinz Erb and his colleagues at the University of Klagenfurt in Vienna created a model of the global agricultural system that forecasts the next 34 years, based on predictions of crop output per hectare, cropland expansion, efficiency of raising livestock, changes in the human diet and other variables. The team reports that given greatly increased crop yields and grazing intensity, global diets could stay much as they are without deforestation. A switch to a vegan or vegetarian diet could, however, allow sufficient expansion of even organically grown crops into former grazing land, without the need to boost yields.

Increased trade between areas of high production and high food demand will be needed to make any of these scenarios feasible.

Nature Commun. 7, 11382 (2016)

\section{ASTRONOMY}

\section{Dwarf dark galaxy leaves smudge}

Astronomers have found an elusive type of miniature galaxy.

Dwarf galaxies formed out of dark matter in the early Universe, but only a small number have been detected. Yashar Hezaveh of Stanford University in California and his colleagues studied images taken by the high-resolution Atacama Large Millimeter/

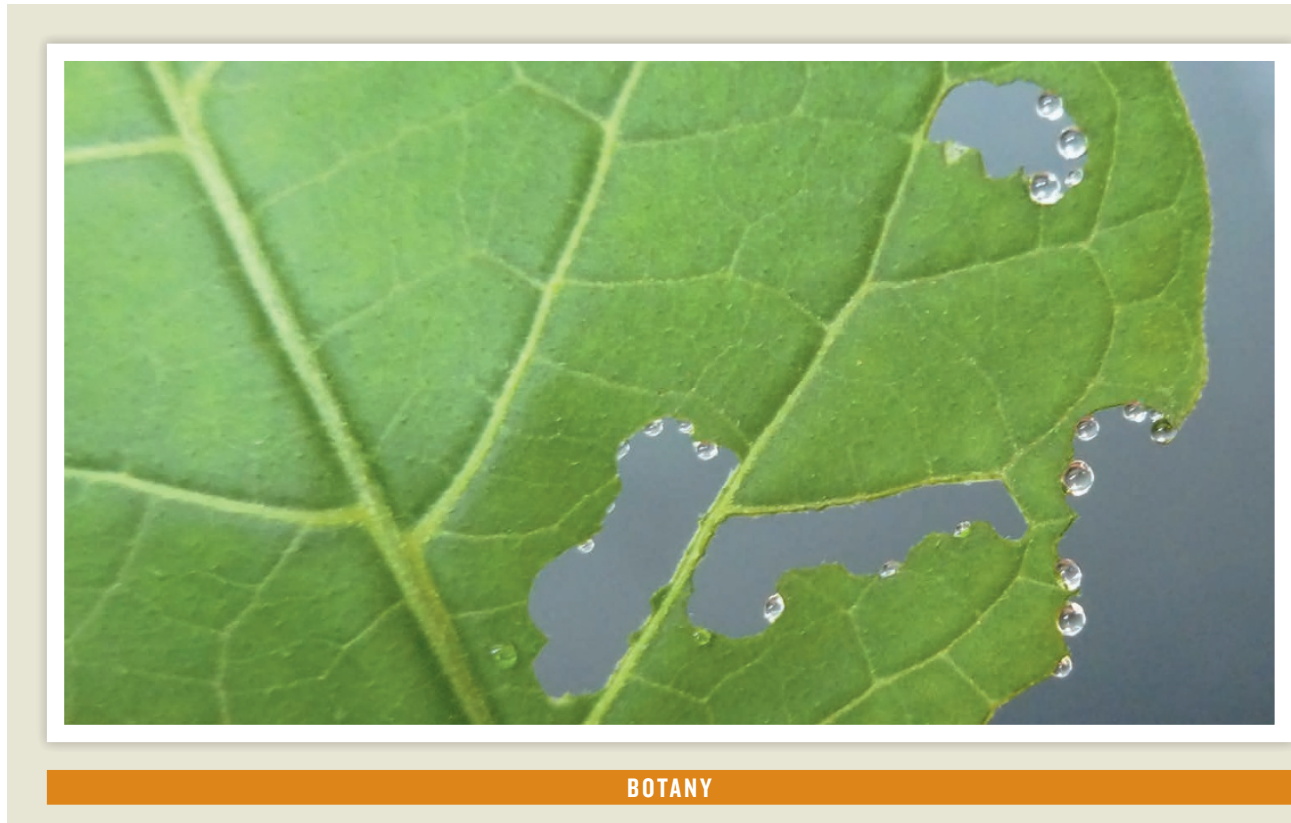

\title{
Plant 'bleeds' nectar from wounds
}

The sugary drops that form on the edges of wounds in a particular plant species have been identified as nectar, which attracts the enemies of leaf-eating pests.

Plants usually heal wounds quickly, but injuries to the bittersweet nightshade (Solanum dulcamara) do not close fully and produce a sugary secretion (pictured). Anke Steppuhn at the Free University of Berlin and her collaborators conducted greenhouse experiments and found that the droplets attracted ants that defended the plant against two herbivorous pests: slugs and flea-beetle larvae.

Other plants produce nectar in specialized organs called nectaries, and the nightshade's organ-free way of making it could represent an evolutionary origin for these organs, the authors suggest.

Nature Plants http://dx.doi.org/10.1038/ nplants.2016.56 (2016) submillimeter Array (ALMA) in Chile. They found a galaxy that was acting like a lens, gravitationally bending light from another, more distant galaxy to form a ring of mirages in the images. The team spotted an additional 'smudge' on these mirages caused by an otherwise invisible dwarf galaxy orbiting the lensing galaxy.

The authors say that ALMA should be able to uncover more dark dwarf galaxies, which would bolster existing models of dark matter.

Astrophys. J. in the press; preprint at http://arxiv.org/ abs/1601.01388 (2016)

\section{NEUROSCIENCE}

\section{Brain may keep watch at night}

Differences in brain activity between the left and right hemispheres may explain why people often sleep poorly in new environments.

Yuka Sasaki at Brown University in Providence, Rhode Island, and her colleagues imaged the brains of people sleeping in an unfamiliar setting, and measured slow-wave activity, a signal associated with nonrapid eye movement (NREM) sleep. During the first night, they found weaker activity in the left hemisphere than in the right - an effect that disappeared on subsequent nights. The researchers also found that on the first night, sounds played to the right ear (which are processed by the left hemisphere) elicited greater brain activation and were more likely to wake the person up than sounds played to the left ear.

The authors speculate that lighter sleep in one hemisphere could have evolved out of a need for vigilance in new environments.

Curr. Biol. http://dx.doi.org/ 10.1016/j.cub.2016.02.063 (2016) 